\title{
ALGEBRAIC OBSTRUCTIONS TO SEQUENTIAL CONVERGENCE IN HAUSDORFF ABELIAN GROUPS
}

\author{
by \\ BRADD CLARK and SHARON CATES \\ University of Southwestern Louisiana \\ (Received June 5, 1996 and in revised form October 10, 1996)
}

\begin{abstract}
Given an abelian group $G$ and a non-trivial sequence in $G$, when will it be possible to construct a Hausdroff topology on $G$ that allows the sequence to converge? As one might expect of such a naive question, the answer is far too complicated for a simple response. The purpose of this paper is to provide some insights to this question, especially for the integers, the rationals, and any abelian groups containing these groups as subgroups. We show that the sequence of squares in the integers cannot converge to 0 in any Hausdroff group topology. We demonstrate that any sequence in the rationals that satisfies a "sparseness" condition will converge to 0 in uncountably many different Hausdorff group topologies.
\end{abstract}

KEY WORDS: Abelian topological group, Hausdorff, sequential convergence

AMS CODE: $22 \mathrm{~A} 05$

\section{INTRODUCTION}

Given an abelian group and a non-trivial sequence in $G$, when will it be possible to construct a Hausdorff group topology on $G$ that allows the sequence to converge? As one might expect of such a naive question, the answer is far too complicated for a simple response. It should be noted that "convergence versus algebra" questions have been studied elsewhere by Frič [2] and others. The purpose of this paper is to provide some insights to this question, especially in certain well-known groups and groups that contain these groups as subgroups.

We shall assume as additional hypothesis throughout the paper that $G$ is an abelian group, and that each sequence under consideration is a one-to-one function from the natural numbers $\mathbb{N}$ into $G$. Since $G$ is a topological group and hence homogeneous, we may, without loss of generality, assume that we wish the sequences under consideration to converge to 0 , the identity element of $G$. Also we shall use the notations $\mathbb{Z}, \mathbb{Q}, \mathbb{R}$, and $S^{1}$ to denote the integers, rationals, reals, and circle group, respectively.

In the course of this paper, we will also need to discuss $\tau_{1} \wedge \tau_{2}$, the "meet" of group topologies $\tau_{1}$ and $\tau_{2}$ on $G$. For any group $G$, the collection of all topologies that transform $G$ into a topological group forms a lattice when set inclusion is used as a partial ordering. As was pointed out in [3], the intersection of two group topologies need not be a group topology. However a tractable meet operator was provided for abelian groups in [1].

\section{SPARSE SEQUENCES}

It is a simple observation that if $g \neq 0$, then the sequence $\langle n g \mid n \in \mathbb{N}\rangle$ cannot converge to 0 in any Hausdorff group topology. The following proposition will allow us to generalize this observation. We say that the set $\left\{g_{1}, g_{2}, \ldots, g_{n}\right\}$ is an $n$-factorization of $g$ if and only if $g=g_{1}+g_{2}+\ldots+g_{n}$. 
PROPOSITION 2.1. If $\left\{x_{n}\right\}_{n \in \mathbb{N}}$ is the image set of a sequence that converges to 0 in $G$ and $\left\{x_{n}\right\}_{n \in \mathbb{N}}$ contains infinitely many pairwise disjoint $n_{0}$-factorizations of $x$ for some fixed natural number $n_{0}$, then $x \in \overline{\{0\}}$.

PROOF. Let $0 \in U$ an open subset of $G$. We can find a neighborhood $V$ of 0 such that $n_{0} V \subseteq U$. Since the sequence converges to 0 , we can find a tail of the sequence in $V$ and hence find an $n_{0}$-factorization of $x$ contained in $V$. Therefore $x \in n_{0} V \subseteq U$, and since $U$ was arbitrary, $x \in \overline{\{0\}}$.

The referee has pointed out that in a Hausdorff topological group proposition 2.1 can be derived by considering sequential convergence as a special case of FLUSH-convergence.

This proposition demonstrates an interesting relationship between topological groups and number theory. If the sequence $\left\langle 1,4,9, \ldots, n^{2}, \ldots\right\rangle$ were to converge to 0 in $\mathbb{Z}$, then the sequence $\left\langle 1,-1,-1,4,-4,-4,9,-9,-9, \ldots, n^{2},-n^{2},-n^{2}, \ldots\right\rangle$ must also converge to 0 . However in [4], it was demonstrated that there are infinitely many pairs of natural numbers $n$ and $m$ satisfying the relationship $n^{2}-2 m^{2}=1$. Hence by the proposition only the indiscrete topology on $\mathbb{Z}$ allows the sequence of squares to converge to 0 .

Now consider the sequence $\left\langle 2,3,5, \ldots, p_{n}, \ldots\right\rangle$ where $p_{n}$ denotes the $n^{\text {th }}$ prime number. If this sequence converges to 0 , then the sequence $\left\langle 2,-2,3,-3,5,-5, \ldots, p_{n},-p_{n_{n} . .}\right\rangle$ must also converge to 0 . Thus by the proposition, if a Hausdorff group topology on $\mathbb{Z}$ allows the sequence to converge to 0 , then the twin prime conjecture is false.

Let $\left\{g_{n}\right\}_{n \in \mathbf{N}}$ be the image set of a sequence in $G$. Then the subgroup $H$ generated by $\left\{g_{n}\right\}_{n \in \mathbb{N}}$ must be a countable subgroup. Since it is trivial in abelian groups to extend topological group structures from a subgroup to all of $G$, it makes sense to study the question of convergence in specific countable subgroups such as $\mathbb{Q}$. As a special case, suppose that $\left\langle x_{k}\right\rangle_{k \in \mathbb{N}}$ is a sequence of positive elements of $\mathbb{Q}$. Without loss of generality, we may assume that the sequence is a strictly inçreasing sequence. We call such a sequence a sparse sequence if and only if:

(1.) $\frac{x_{k+1}}{x_{k}}>\frac{k^{2}+3 k}{2(k+1)}$ for every $k \in \mathbb{N}$ and

(2.) $\frac{x_{k+1}}{x_{k}}>\frac{k^{2}+2 k}{k+1}$ for infinitely many $k \in \mathbb{N}$.

PROPOSITION 2.2. If $\left\langle x_{k}\right\rangle_{k \in \mathbb{N}}$ is a sparse sequence in $\mathbb{Q}$, then there exist uncountably many Hausdorff group topologies on $\mathbb{Q}$ that have $\left\langle x_{k}\right\rangle_{k \in \mathbf{N}}$ converge to 0 .

PROOF. For any real number $r$, we can define a function $f_{r}: \mathbb{R} \rightarrow S^{1}$ by $f_{r}(x)=r x$. If we place the Euclidean topology on $S^{1}$, we can use $f_{r}$ to place a group topology on $\mathbb{R}$. Let $U_{k}=\left(-\frac{1}{k}, \frac{1}{k}\right)$ in $S^{1}$. Then since $\left\{U_{k}\right\}_{k \in \mathrm{N}}$ is a fundamental system for the topology on $S^{1}$, $\left\{f_{r}^{-1}\left(U_{k}\right)\right\}_{k \in \mathbb{N}}$ is a fundamental system for a group topology on $\mathbb{R}$. While none of these topologies are Hausdorff topologies, we note that whenever $r$ is an irrational number, $\left.f_{r}\right|_{Q}$ is one-to-one, hence, in this situation the relative topology on $\mathbb{Q}$ generated by $f_{r}$ will be a Hausdorff group topology.

Let $U^{k}=f_{r}^{-1}\left(U_{k}\right)$. If $x_{k} \in U^{k}$, it is necessary for $r x_{k} \in U_{k}$. But this means that either $m \leq r x_{k}<m+\frac{1}{k}$ or $(m-1)+\frac{k-1}{k}<r x_{k} \leq m$ for some $m \in \mathbb{Z}$. Therefore, we must have that either $\frac{m}{x_{k}} \leq r<\frac{m k+1}{k x_{k}}$ or $\frac{m k-1}{k x_{k}}<r \leq \frac{m}{x_{k}}$. 
Hence for $x_{k} \in U^{k}$, it is necessary that $r \in \cup_{m \in \mathbf{Z}}\left(\frac{m k-1}{k x_{k}}, \frac{m k+1}{k x_{k}}\right)=V^{k} \subseteq \mathbb{R}$. We wish to show that given an interval from $V^{k}$, say $\left(\frac{m k-1}{k x_{k}}, \frac{m k+1}{k x_{k}}\right)$, there exists an integer $m_{0}$ such that $\left(\frac{m_{0}(k+1)-1}{(k+1) x_{k+1}}, \frac{m_{0}(k+1)+1}{(k+1) x_{k+1}}\right) \subsetneq\left(\frac{m k-1}{k x_{k}}, \frac{m k+1}{k x_{k}}\right)$. For this to happen, it is necessary and sufficient for $\frac{m k-1}{k x_{k}}<\frac{m_{0}(k+1)-1}{(k+1) x_{k+1}}$ and $\frac{m_{0}(k+1)+1}{(k+1) x_{k+1}}<\frac{m k+1}{k x_{k}}$, or equivalently that

$$
\frac{(m k-1) x_{k+1}}{k x_{k}}+\frac{1}{k+1}<m_{0}<\frac{(m k+1) x_{k+1}}{k x_{k}}-\frac{1}{k+1} .
$$

Since $\left\langle x_{k}\right\rangle_{k \in \mathbf{N}}$ is sparse, we have that:

$$
\frac{(m k+1) x_{k+1}}{k x_{k}}-\frac{(m k-1) x_{k+1}}{k x_{k}}-\frac{2}{k+1}>\frac{(m k+1)\left(k^{2}+3 k\right)}{2 k(k+1)}-\frac{(m k-1)\left(k^{2}+3 k\right)}{2 k(k+1)}-\frac{2}{k+1}=1 .
$$

Thus each interval used in forming $V^{k}$ contains at least one full interval from the construction of $V^{k+1}$.

Now infinitely often we have that $\frac{x_{k+1}}{x_{k}}>\frac{k^{2}+2 k}{k+1}$. Using a similar argument to the above, we can show that for infinitely many $k$, every interval in $V^{k}$ contains two intervals from $V^{k+1}$. But this means that $\cap_{k=1}^{\infty} V^{k}$ contains a Cantor set and hence is uncountable. Therefore, there exist uncountably many irrational numbers $r$ that generate Hausdorff group topologies on $\mathbb{Q}$ that have the sequence $\left\langle x_{k}\right\rangle_{k \in \mathrm{N}}$ converging to 0 .

If $\left\{x_{n}\right\}_{n \in \mathbb{N}}$ is the image set of a sequence in $\mathbb{Q}$, we can decompose $\left\{x_{n}\right\}_{n \in \mathbb{N}}$ into two subsets, $\left\{y_{z}\right\}_{i \in \mathbf{N}}$ and $\left\{z_{j}\right\}_{j \in \mathbf{N}}$ where each $y_{2} \geq 0$ and each $z_{j}<0$. Following a rearrangement of terms, we can assume that $y_{\imath+1}>y_{\imath}$ and $z_{\jmath+1}<z$ for all $i, j \in \mathbb{N}$.

COROLLARY 2.3. If $\left\langle x_{n}\right\rangle_{n \in \mathbb{N}}$ is a sequence in $\mathbb{Q}$ with corresponding sequences $\left\langle y_{\imath}\right\rangle_{\imath \in \mathbb{N}}$ and $\left\langle\left|z_{j}\right|\right\rangle_{j \in \mathbb{N}}$ and $\left\{y_{2}\right\}_{\imath \in \mathbf{N}} \cup\left\{\left|z_{\jmath}\right|\right\}_{\jmath_{j} \in \mathbf{N}}$ is the image of a sparse sequence, then there are uncountably many Hausdorff group topologies on $\mathbb{Q}$ which have $\left\langle x_{n}\right\rangle_{n \in \mathbb{N}}$ converge to 0 .

The method that we have used to construct group topologies on $\mathbb{Q}$ has its limitations. If we use it to create group topologies $\tau_{1}$ and $\tau_{2}$ in which the sparse sequences $\left\langle x_{2}\right\rangle_{2 \in \mathbb{N}}$ and $\left\langle y_{2}\right\rangle_{2 \in \mathbb{N}}$ converge to 0 , then we can be sure that the combined sequence $\left\langle x_{1}, y_{1}, x_{2}, y_{2}, x_{3}, y_{3}, \ldots\right\rangle$ will converge to 0 in $\tau_{1} \wedge \tau_{2}$. However by [1], we know that $\left\{\frac{n}{r_{1}}+\frac{m}{r_{2}}+(-\epsilon, \epsilon) \mid n, m \in \mathbb{Z}\right.$ and $\left.\epsilon>0\right\}$ is a fundamental system for $\tau_{1} \wedge \tau_{2}$ where $r_{1}$ and $r_{2}$ are the irrationals associated with the topologies $\tau_{1}$ and $\tau_{2}$ respectively. Now $\tau_{1} \wedge \tau_{2}$ will be a Hausdorff topology if and only if $\frac{r_{1}}{r_{2}} \in \mathbb{Q}$, and will be the indiscrete topology otherwise.

Suppose that $H<G$ and that $\phi: H \rightarrow \mathbb{Q}$ is an isomorphism into $\mathbb{Q}$. We say that a sequence $\left\langle x_{n}\right\rangle_{n \in \mathbb{N}}$ in $H$ is a sparse in $H$ if and only if $\left\langle\phi\left(x_{n}\right)\right\rangle_{n \in \mathbb{N}}$ is sparse in $\mathbb{Q}$.

PROPOSITION 2.4. Let $H<G$ be a direct sum of cyclic groups and groups isomorphic to $\mathbb{Q}$. If $\left\langle x_{n}\right\rangle_{n \in \mathbb{N}}$ is a sequence in $H$ whose associated coordinate sequences are either sparse or eventually 0 , then there is a Hausdorff group topology on $G$ that has $\left\langle x_{n}\right\rangle_{n \in \mathrm{N}}$ converging to 0 .

ACKNOWLEDGEMENT. The authors are grateful to the referee for his suggestions in the preparation of this paper. 
B. CLARK AND S. CATES

\section{REFERENCES}

[1] B. Clark and V. Schneider, "The meet operator in the lattice of group topologies," Canad. Math. Bull. 29 (1986), 478-481.

[2] R. Frič, "Convergence and numbers," Topology Appl. 70 (1996), 139-146.

[3] P. Samuel, "Ultrafilters and compactifications of uniform spaces," Trans. Amer. Math. Soc. 64 (1948), 100-134.

[4] D. Shanks, "Solved and Unsolved Problems in Number Theory," Second Edition, Chelsea Publishing Company, New York, 1978. 


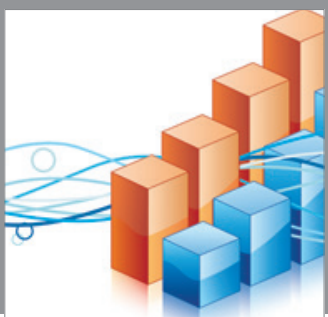

Advances in

Operations Research

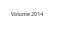

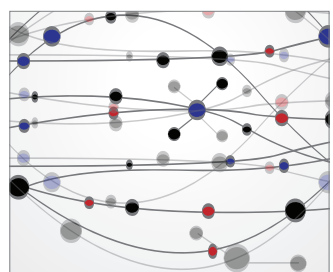

\section{The Scientific} World Journal
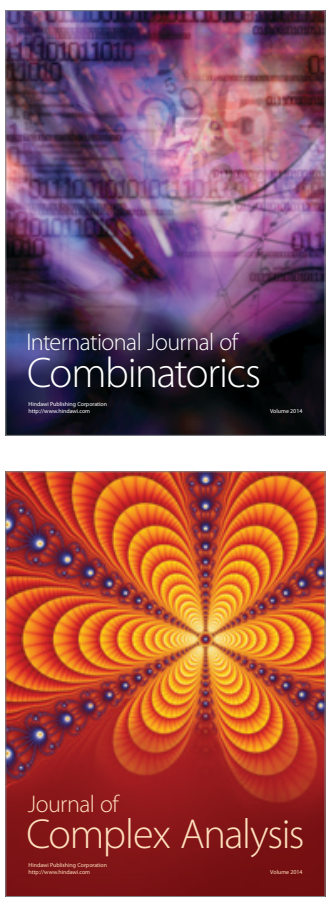

International Journal of

Mathematics and

Mathematical

Sciences
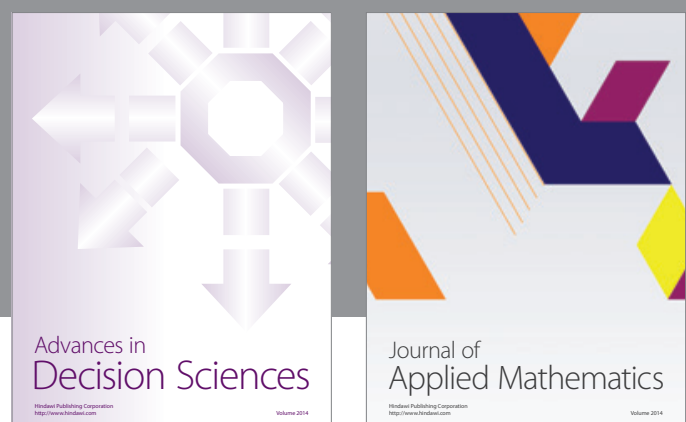

Journal of

Applied Mathematics
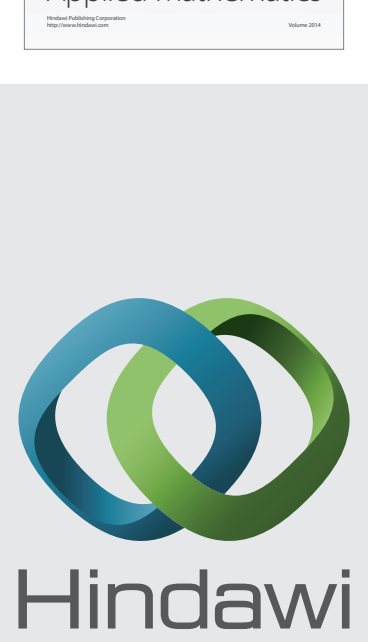

Submit your manuscripts at http://www.hindawi.com
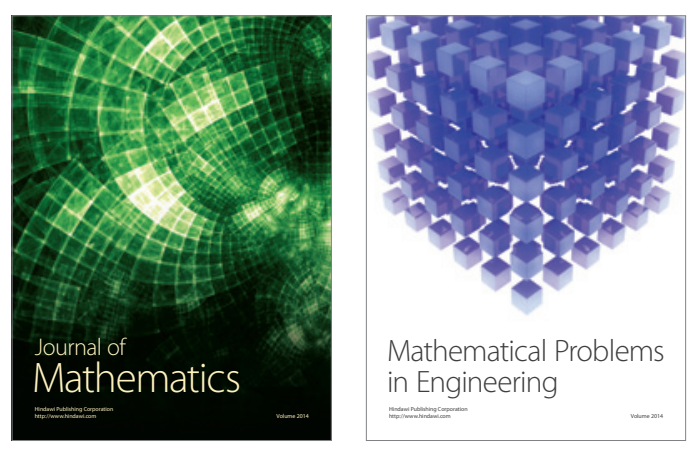

Mathematical Problems in Engineering
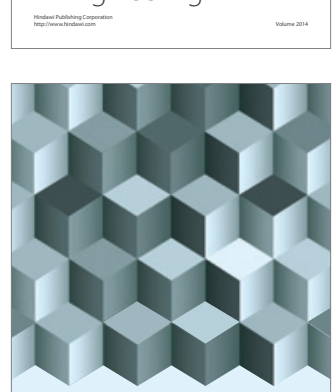

Journal of

Function Spaces
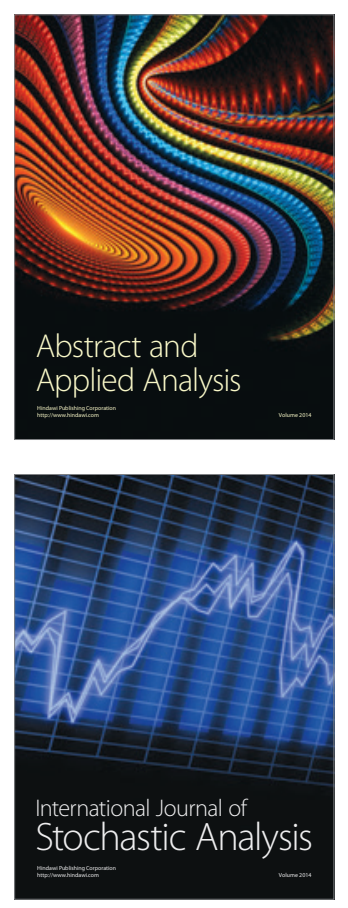

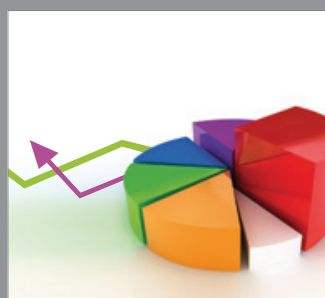

ournal of

Probability and Statistics

Promensencen
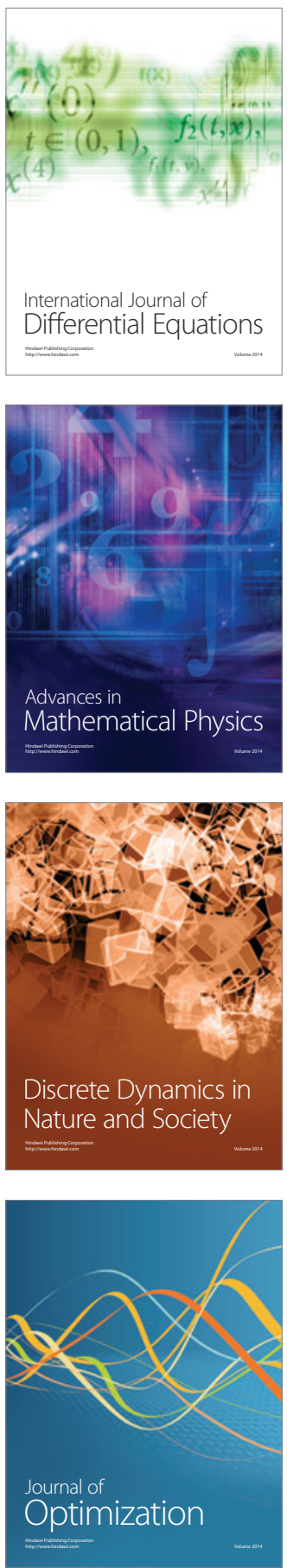